\title{
A case of acute kidney injury with vancomycin and piperacillin/tazobactam
}

\begin{abstract}
Background: Acute kidney injury can be attributed to several causes, including druginduced nephrotoxicity. A dramatic and acute increase in serum creatinine has been observed in a patient receiving vancomycin and piperacillin/tazobactam therapy. Documentation of potential kidney injury during concomitant therapy with these two agents is found in current literature.
\end{abstract}

Objective: To describe a patient case in which the concomitant use of vancomycin and piperacillin/tazobactam resulted in acute kidney injury.

Design: Case Report

Setting: A 522-bed community hospital.

Patient: A Hispanic female with normal renal function presents with osteomyelitis in both feet. During therapy with vancomycin and piperacillin/tazobactam administrations, acute and significant increases in serum creatinine were observed.

Intervention: Discontinuation of vancomycin and administration of acetylcysteine

Measurements: Serum creatinine levels and timing of offending agents as well as the doses (vancomycin, piperacillin/tazobactam) were all documented.

Results: Serum creatinine levels of the patient increased from $1.0 \mathrm{mg} / \mathrm{dL}$ up to $5.1 \mathrm{mg}$ / $\mathrm{dL}$ over the three days with three total doses of vancomycin and 2 to 3 doses of piperacillin/tazobactam each day. The increase in serum creatinine correlated with the concomitant administration of these two agents.

Conclusion: Patients receiving both vancomycin and pipercillin/tazobactam require close monitoring for signs of acute kidney injury. While some risk factors have been proposed in literature, further research is needed to further define these risk factors and time to acute kidney injury with these medications.

Keywords: vancomycin, piperacillin/tazobactam, nephrotoxicty
Volume 7 Issue 5 - 2017

\author{
Pamella S Ochoa, Brian T Terrell \\ Texas Tech University Health Sciences Center- School of \\ Pharmacy, USA
}

Correspondence: Pamella S Ochoa, Texas Tech University
Health Sciences Center- School of Pharmacy, USA, Email pamella.ochoa@ttuhsc.edu Received: November 03, 2017 | Published: November 17,
2017

\section{Background}

Acute kidney injury can result from a multitude of factors, including drug-induced causes. Medications like vancomycin and piperacillin/tazobactam have shown to be correlated with deterioration of the kidneys when used in excess or concomitantly with other potential offending agents. ${ }^{1,2}$ While these agents have been both been associated with potential nephrotoxcity there is limited data on the exact mechanisms and roles in potential kidney injury. Further, no patient case reports describing kidney injury with concomitant use of vancomycin and pipercillin/tazobactam is found in current literature that describes the series of events leading to acute kidney injury.

Although there is no known mechanism in which vancomycin may induce kidney injury, current animal data suggests that it has oxidative effects on cells of the proximal renal tubule. ${ }^{3}$ The primary mechanism thus appears to be necrosis due to renal tubular ischemia secondary to oxidative effects of vancomycin. ${ }^{3}$ In addition to oxidative stress and resultant mitochondrial damage, contributions from the complement pathway and inflammation have been postulated. Evidence of tissue repair have also been detected in addition to necrosis in animal studies. ${ }^{4}$ The most common risk factor for vancomycin-associated nephrotoxicity is an increased serum trough concentrations. ${ }^{5}$ Various studies have found an increased risk of nephrotoxicity as trough concentrations increase, with trough concentration greater than 15 milligrams/liter ( $\mathrm{mg} / \mathrm{L}$ ) showing a moderate to high risk, while a trough concentration greater than $20 \mathrm{mg} /$ liter showed a much greater risk. ${ }^{5}$ This vancomycin-associated nephrotoxicity may be reversible in some patients with studies revealing that renal function may return to baseline or below predefined toxicity thresholds within a week or less following discontinuation of vancomycin. ${ }^{5}$

Piperacillin/tazobactam, the other offending agent of concern, may induce kidney injury by causing interstitial nephritis (AIN). ${ }^{6}$ Druginduced AIN (DIAIN) is a known cause of impaired renal function and deterioration especially with antibiotics such as piperacillin/ tazobactam and non-steroidal anti-inflammatory drugs (NSAIDs). ${ }^{7}$ AIN is defined as an increased levels of inflammatory infiltrates such as lymphocytes (mostly $\mathrm{T}$ cells), macrophages, eosinophils, and plasma cells as well as edema within the renal tubules and interstitium which is usually associated with renal dysfunction. ${ }^{6,7}$ The pathogenesis of DIAIN is believed to be on an immunologic basis such as drug components interacting with endogenous antigens of tubular basement membrane (TBM), as evidenced by the common appearance of extrarenal hypersensitivity reactions. ${ }^{7}$ It is suggested that piperacillin/tazobactam can bind to normal components of TBM or can mimic the actions of the normal antigen present in TBM 
to induce an immunologic response, like developing anti-TBM antibodies, subsequently leading to excess production and recruitment of inflammatory infiltrates. ${ }^{7}$

Although there is some data on mechanisms of nephrotoxicity for each of agents above, there is limited data of the synergistic nephrotoxicity caused by the combination of the two antibiotics. One study comparing the use of vancomycin with piperacillin/tazobactam and vancomycin with cefepime found higher incidence of acute renal failure in patients given both vancomycin and piperacillin/ tazobactam than with cefepime; however, no statistical significance was demonstrated. ${ }^{8}$ Because both antibiotics are eliminated through renal mechanisms and show nephrotoxicity individually, it is predicted that the concomitant use of vancomycin and piperacillin/ tazobactam can cause a higher incident of nephrotoxicity than when used as monotherapy. ${ }^{1,2,8}$

Nephrotoxicity can be defined as patients who developed acute renal failure. Acute renal failure can be defined as serum creatinine (SCr) increase by $0.5 \mathrm{mg} / \mathrm{dL}$ or increase by $50 \%$ from baseline. ${ }^{8}$ In a person without any kidney disease, serum creatinine is constant with very small variability. ${ }^{9}$ However, serum creatinine tends to increase in compromised kidneys and glomerulus filtration rate declines. ${ }^{9}$ Although there are many medications that have shown to be directly correlated with nephrotoxicity, there has yet to be sufficient reports of piperacillin/tazobactam, when used concomitantly with vancomycin, to augment the effects of nephrotoxicity.

\section{Case report}

A 40-year-old, $60 \mathrm{in}, 70.7 \mathrm{~kg}$, Hispanic female was transferred from an outside hospital for a higher level of care for an initial complaint of a left foot infection. She had a long-standing history of type-1 diabetes mellitus, hypertension and diabetic peripheral neuropathy. Her home medication regimen prior to admission included amitriptyline 25mg PO daily, lisinopril/hydrochlorothiazide 20/12.5mg PO daily and Novolin $\mathbb{R}$ 70/30: 50units subcutaneously twice daily. At the outside hospital the patient had been receiving vancomycin and levofloxacin (dose not mentioned in H\&P) and the infection had not improved. Upon admission, the patient's creatinine was reported at $1.0 \mathrm{mg} / \mathrm{dL}$. Scheduled medications started on the date of admission include enoxaparin $40 \mathrm{mg}$ subcutaneously daily, insulin glargine 20units subcutaneously twice daily, amlodipine $5 \mathrm{mg}$ PO daily, hydrochlorothiazide $12.5 \mathrm{mg}$ PO daily, lisinopril 10mg PO twice daily, insulin lispro 8 units subcutaneously three times daily with meals and sliding scale, ascorbic acid 500mg PO daily, zinc sulfate $220 \mathrm{mg}$ PO daily, amitriptyline $25 \mathrm{mg}$ PO at bedtime, ferrous sulfate $325 \mathrm{mg}$ PO twice daily and as needed medication use included oxycodone $5 \mathrm{mg}$ PO daily. Specifically for the infection, the patient was started on empiric coverage with a vancomycin $1250 \mathrm{mg}$ IV one time dose as well as piperacillin/tazobactam $3.375 \mathrm{~g}$ IV q $6 \mathrm{hrs}$. After receiving one dose of vancomycin and two doses of piperacillin/tazobactam the patient's creatinine increased to $1.5 \mathrm{mg} / \mathrm{dL}$. On this same day, after labs were obtained, the patient received contrast dye for a CT of the foot to rule out osteomyelitis. The next day, after another dose of vancomycin $1000 \mathrm{mg}$ IV every 24 hours and three doses of piperacillin/ tazobactam, the patient's creatinine increased to $2.6 \mathrm{mg} / \mathrm{dL}$ and on this day the patient was taken into surgery for irrigation and debridement of confirmed osteomyelitis of both feet. The patient did receive one more dose of vancomycin on this day and four doses of piperacillin/ tazobactam. The next creatinine returned at $3.3 \mathrm{mg} / \mathrm{dL}$ and a random vancomycin concentration eight hours after the dose was measured at $45.5 \mathrm{mcg} / \mathrm{mL}$. On this day, vancomycin was discontinued and the patient was started on daptomycin $6 \mathrm{mg} / \mathrm{kg}$ IV every 24 hours, while piperacillin/tazobactam was continued until the evening dose but then held for two days. Surgical cultures at this point had identified MRSA as the main organism of infection but the piperacillin/tazobactam was considered for restart secondary to concern of polymicrobial infection. The next day, morning labs revealed that the patient's creatinine continued to rise to $4.5 \mathrm{mg} / \mathrm{dL}$. At this point, hydration was intensified. The following day the patient's creatinine continued to rise to $5.1 \mathrm{mg} / \mathrm{dL}$. At this point, the piperacillin/tazobactam dose was restarted at $2.25 \mathrm{~g}$ IV every 8hours and the patient was given a onetime dose of oral acetylcysteine to potentially help with renal function. Over the next few days, the patient's creatinine trend was as follows: $4.7 \mathrm{mg} / \mathrm{dL}, 3.5 \mathrm{mg} / \mathrm{dL}, 3.0 \mathrm{mg} / \mathrm{dL}, 2.8 \mathrm{mg} / \mathrm{dL}$ and $2.3 \mathrm{mg} / \mathrm{dL}$. Figure 1 illustrates the relationship between administration of the perceived agents that caused and potentiated the nephrotoxicity and the patient's creatinine. Figure 1 represents the trend of creatinine for this patient before during and after the preceding events. The patient subsequently continued to have slightly impaired renal function but continued to improve and was transferred to an extended care unit for long-term antibiotic therapy. The patient was eventually discharged home from the hospital two months later.

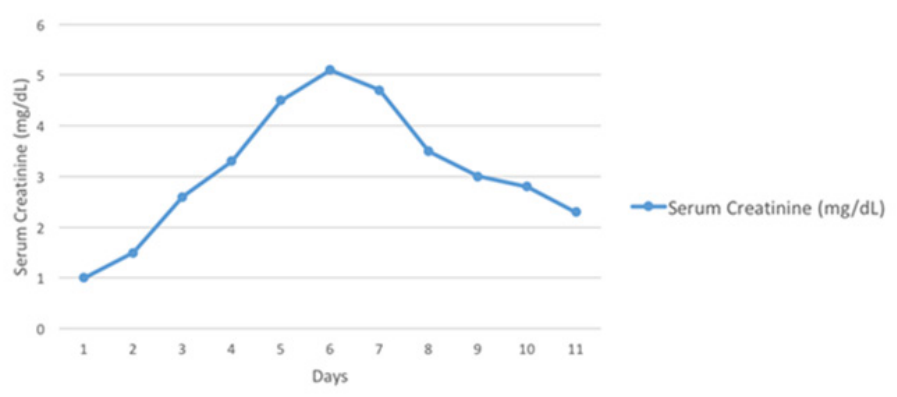

Figure I Serum Creatine over course of hospital admission.

\section{Discussion}

This case report was chosen to be reported as it was unclear which potentially nephrotoxic agent was the cause of the large increase in creatinine. The patient had tolerated vancomycin at the outside hospital and had a normal creatinine when she arrived at the hospital. The timeline then becomes interesting, as there was a $0.5 \mathrm{mg} / \mathrm{dL}$ jump in the measure for renal function after the addition of piperacillin/ tazobactam but before the administration of IV contrast dye. While vancomycin and contrast dye are known to potentially cause renal toxicity, piperacillin/tazobactam does not have a lot of data regarding renal toxicity associated with it in normal renal function patients but some literature does exist. ${ }^{10-14}$ It is interesting that this specific combination of agents may be a sort of "perfect storm" in which the different mechanisms add insult to injury and result in additive nephrotoxicity particular piperacillin/tazobactam potentially increasing the time to renal recovery. ${ }^{13}$ While it possible that the nephrotoxicity had begun before administration of these agents the timeline of rise in serum creatinine implicates that these agents at least played some role. The continued rise in serum creatinine after discontinuation of vancomycin but continuation of piperacillin/ tazobactam is cause to consider discontinuation of both of these medications if acute kidney injury is identified (Figure 2).

Figure 2 Serum Creatine and timings of agents. 


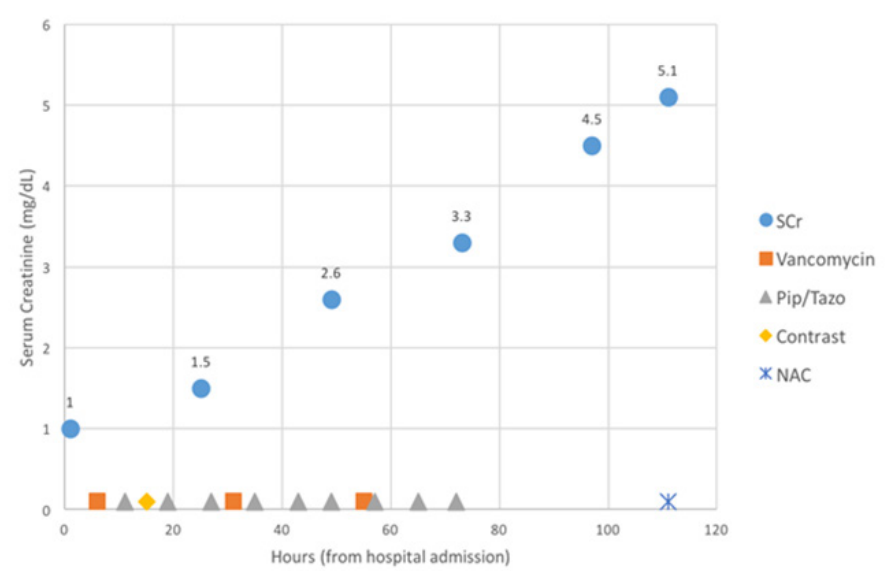

Figure 2 Serum Creatine and timings of agents.

\section{Conclusion}

This case report shows that there may be additive nephrotoxic effects of piperacillin/tazobactam when it is added to vancomycin. While the large jump in creatinine may be also attributed to IV contrast dye, the timeline lends itself to the conclusion that this combination should at least lead to increased monitoring of renal function in patients in which it is initiated.

\section{Acknowledgements}

We would like to acknowledge Khumphan Amaratana for his contribution toward the revision of the manuscript.

\section{Conflict of interest}

The author declares no conflict of interest.

\section{References}

1. Luther MK, Timbrook TT, Caffrey AR, et al. Vancomycin plus piperacillin-tazobactam and acute kidney injury in adults:a systematic review and meta-analysis. Crit Care Med. 2017.

2. Kim T, Kandiah S, Patel M, et al. Risk factors for kidney injury during vancomycin and piperacillin/tazobactam administration, including increased odds of injury with combination therapy. BMC Res Notes. 2015;8:579.
3. Elyasi S, Khalili H, Dashti-khavidaki S, et al. Vancomycin-induced nephrotoxicity: mechanism, incidence, risk factors and special populations. A literature review. Eur J Clin Pharmacol. 2012;68(9):1243-1255.

4. Gupta A, Biyani M, Khaira A. Vancomycin nephrotoxicity: myths and facts. Neth J Med. 2011;69(9):379-383.

5. van Hal SJ, Paterson DL, Lodise TP. Systematic review and meta-analysis of vancomycin-induced nephrotoxicity associated with dosing schedules that maintain troughs between 15 and 20 milligrams per liter. Antimicrob Agents Chemother. 2013;57(2):734-744.

6. Taber SS, Mueller BA. Drug-Associated Renal Dysfunction. Crit Care Clin. 2006;22(2):357-374.

7.Praga M, Gonzalez E. Acute interstitial nephritis. Kidney Int. 2010;77(11):956-961.

8. Moenster RP, Linneman TW, Finnegan PM, et al. Acute renal failure associated with vancomycin and beta-lactams for the treatment of osteomyelitis in diabetics: piperacillin/tazobactam compared to cefepime. Clin Microbiol Infect. 2013;20(6):O384-0389.

9. Traynor J, Mactier R, Geddes CC, et al. How to measure renal function in clinical practice. BMJ. 2006;333(7571):733-737.

10. Parfrey PS, Griffiths SM, Barrett BJ, et al. Contrast material induced renal failure in patients with diabetes mellitus, renal insufficiency, or both. A prospective controlled study. $N$ Engl J Med. 1989;320(3):143-149.

11. Lodise TP, Lomaestro B, Graves J, et al. Larger vancomycin doses (at least four grams per day) are associated with an increased incidence of nephrotoxicity. Antimicrob. Agents Chemother. 2008;52(4):1330-1336.

12. Lodise TP, Patel N, Lomaestro BM, et al. Relationship between initial vancomycin concentration-time profile and nephrotoxicity among hospitalized patients. Clin Infect Dis. 2009;49(4):507-514.

13. Jensen J-US, Hein L, Lundgren B, Bestle MH, et al. Kidney failure related to broad-spectrum antibiotics in critically ill patients:secondary end point results from a 1200 patient randomised trial. BMJ Open. 2012;2:e00635.

14. Wyeth Pharmaceuticals Inc. Zosyn ${ }^{\circledR}$ package insert. Philadelphia; 2012. 